\title{
$L$-values and the Fitting ideal of the tame kernel for relative quadratic extensions
}

\author{
by \\ Jonathan W. Sands (Burlington, VT)
}

1. Introduction. Let $E / F$ be a fixed relative quadratic extension of algebraic number fields with Galois group $G=\langle\tau\rangle$. We also fix a finite set $S$ of primes of $F$ which contains all of the infinite primes of $F$ and all of the primes which ramify in E. Associated with this data is a Stickelberger function, or equivariant $L$-function, $\theta_{E / F}^{S}(s)$. It is a meromorphic function of $s$ with values in the group ring $\mathbb{C}[G]$. To define it, let $\mathfrak{p}$ run through the (finite) primes of $F$ not in $S$, and $\mathfrak{a}$ run through integral ideals of $F$ which are relatively prime to each of the elements of $S$. Then $\mathrm{Na}$ denotes the absolute norm of the ideal $\mathfrak{a}, \sigma_{\mathfrak{a}} \in G$ is the well-defined automorphism attached to $\mathfrak{a}$ via the Artin map, and

$$
\theta_{E / F}^{S}(s)=\sum_{\substack{\mathfrak{a} \text { integral } \\(\mathfrak{a}, S)=1}} \frac{1}{\mathrm{Na}^{s}} \sigma_{\mathfrak{a}}^{-1}=\prod_{\text {prime } \mathfrak{p} \notin S}\left(1-\frac{1}{\mathrm{~Np}^{s}} \sigma_{\mathfrak{p}}^{-1}\right)^{-1} .
$$

These expressions converge for the real part of $s$ greater than 1 and the function they define extends meromorphically to all of $\mathbb{C}$.

The function $\theta_{E / F}^{S}(s)$ is connected with the arithmetic of the number fields $E$ and $F$ in ways one would like to make as precise as possible. Define the ring of $S$-integers $\mathcal{O}_{F}^{S}$ of $F$ to be the set of elements of $F$ whose valuation is non-negative at every prime not in $S$. Similarly, define the $\operatorname{ring} \mathcal{O}_{E}^{S}$ of $S$-integers of $E$ to be the set of elements of $E$ whose valuation is nonnegative at every prime not in $S_{E}$, the set of all primes of $E$ which lie above some prime in $S$. The zeta-functions defined by $\zeta_{F}^{S}(s) \sigma_{\mathcal{O}_{F}}=\theta_{F / F}^{S}(s)$ and $\zeta_{E}^{S}(s) \sigma_{\mathcal{O}_{E}}=\theta_{E / E}^{S_{E}}(s)$ may be viewed as the zeta-functions of the Dedekind domains $\mathcal{O}_{F}^{S}$ and $\mathcal{O}_{E}^{S}$.

2000 Mathematics Subject Classification: Primary 11R42; Secondary 11R70, 19F27.

Key words and phrases: tame kernel, Stickelberger ideal, relative quadratic extension, Fitting ideal, Artin $L$-function. 
Our focus will be on the "higher Stickelberger element" $\theta_{E / F}^{S}(-1)$. It is conjecturally related to the algebraic $K$-group $K_{2}\left(\mathcal{O}_{E}^{S}\right)$. This group is known to be finite by [4] and [11], and called the tame kernel of $E$.

The precise statement of the conjectured arithmetic interpretation of $\theta_{E / F}^{S}(-1)$ will also involve another finite group. Let $\mu_{\infty}$ denote the group of all roots of unity in an algebraic closure $\overline{\mathbb{Q}}$ of $\mathbb{Q}$ containing $E$, and let $\mathcal{G}$ denote the Galois group of $\overline{\mathbb{Q}} / \mathbb{Q}$. Define $W_{2}=W_{2}(\overline{\mathbb{Q}})$ to be the $\mathbb{Z}[\mathcal{G}]$-module whose underlying group is $\mu_{\infty}$, with the action of $\gamma \in \mathcal{G}$ on $\omega \in W_{2}$ given by $\omega^{\gamma}=\gamma^{2}(\omega)$. Then for any subfield $L$ of $\overline{\mathbb{Q}}$, let $W_{2}(L)$ be the submodule fixed under this action by the Galois group of $\overline{\mathbb{Q}}$ over $L$. Then $W_{2}(E)$ naturally becomes a $\mathbb{Z}[G]$-module, where the action of $G$ arises by lifting elements of $G$ to the Galois group of $\overline{\mathbb{Q}}$ over $F$. One easily sees that the $G$-fixed submodule $W_{2}(E)^{G}$ equals $W_{2}(F)$. We use the notation $w_{2}(L)=\left|W_{2}(L)\right|$, which we note is finite for any algebraic number field $L$.

The conjecture of Birch and Tate (see Section 4 of [14]) gives a precise arithmetic interpretation of $\zeta_{F}^{S}(-1)$. We state an extended form of it for arbitrary finite $S$ which is an easy consequence of the original conjecture for minimal $S$ (see Corollary 3.3 of [12]).

Conjecture 1.1 (Extended Birch-Tate conjecture). Suppose that F is totally real. Then

$$
\zeta_{F}^{S}(-1)=(-1)^{|S|} \frac{\left|K_{2}\left(\mathcal{O}_{F}^{S}\right)\right|}{w_{2}(F)} .
$$

Deep results on Iwasawa's main conjecture in [10] and [15] lead to the following (see [8]).

ThEOREM 1.2. The Birch-Tate conjecture holds if $F$ is abelian over $\mathbb{Q}$, and the odd part holds for all totally real $F$.

The 2-part of the Birch-Tate conjecture for $F$ would follow from the 2-part of Iwasawa's main conjecture for $F$. Our main result also involves the annihilator $\operatorname{Ann}_{\mathbb{Z}[G]}\left(W_{2}(E)\right)$ of $W_{2}(E)$ in $\mathbb{Z}[G]$.

THEOREM 1.3. Let $E / F$ be a relative quadratic extension of totally real number fields, with Galois group G. Assume that the 2-part of the BirchTate conjecture holds for $E$ and for $F$. Then the (first) Fitting ideal of $K_{2}\left(\mathcal{O}_{E}^{S}\right)$ as a $\mathbb{Z}[G]$-module is

$$
\operatorname{Fit}_{\mathbb{Z}[G]}\left(K_{2}\left(\mathcal{O}_{E}^{S}\right)\right)=\operatorname{Ann}_{\mathbb{Z}[G]}\left(W_{2}(E)\right) \theta_{E / F}^{S}(-1) .
$$

Equality of the extension of these ideals to $\mathbb{Z}[1 / 2][G]$ holds unconditionally.

This result is to be compared with results computing the Fitting ideals of certain modified ideal class groups in terms of more classical Stickelberger ideals. Notable among these is the recent result of Greither [6] identifying 
the Fitting ideal of the dual of the odd part of the minus part of the ideal class group of a CM field which is abelian over a totally real field. Assuming the equivariant Tamagawa number conjecture, this ideal is shown to equal a Stickelberger ideal obtained from the values at $s=0$ of Stickelberger functions for subextensions. Our Theorem 1.3 begins to provide an analog at $s=-1$. The comparison can provide some insight since the odd part in Theorem 1.3 is unconditional, and the result on the 2-part, though conditional, has no counterpart as yet at $s=0$.

For ease of notation, from now on, let $\mathcal{R}=\mathbb{Z}[G]$. Denote the Fitting ideal we are interested in by $\mathcal{I}^{\text {Fit }}=\operatorname{Fit}_{\mathcal{R}}\left(K_{2}\left(\mathcal{O}_{E}^{S}\right)\right)$, and the generalized Stickelberger ideal by $\mathcal{I}^{\text {Sti }}=\operatorname{Ann}_{\mathcal{R}}\left(W_{2}(E)\right) \theta_{E / F}^{S}(-1)$. The ingredients we will need to prove their equality are cohomology computations, properties of Fitting ideals, and some information on the ideal structure of $\mathcal{R}$ and related rings.

2. Cohomology of $K_{2}\left(\mathcal{O}_{E}^{S}\right)$. We continue to assume throughout that $E / F$ is a relative quadratic extension of totally real fields with Galois group $G=\langle\tau\rangle$. For a $\mathbb{Z}[G]$-module $M$, we define $M^{G}$ as usual to be the submodule of $M$ which is annihilated by $1-\tau$, and $M^{-}$to be the submodule annihilated by $1+\tau$. Also let $M_{G}$ denote the module of co-invariants of $M$ under the action of $G$. The following result provides the key to computing the orders we will need.

THEOREM 2.1. Under our assumptions, the transfer map from $K_{2}\left(\mathcal{O}_{E}^{S}\right)$ to $K_{2}\left(\mathcal{O}_{F}^{S}\right)$ is surjective with kernel $K_{2}\left(\mathcal{O}_{E}^{S}\right)^{1-\tau}$. So we have a short exact sequence

$$
0 \rightarrow K_{2}\left(\mathcal{O}_{E}^{S}\right)^{1-\tau} \rightarrow K_{2}\left(\mathcal{O}_{E}^{S}\right) \stackrel{\text { Trans }}{\longrightarrow} K_{2}\left(\mathcal{O}_{F}^{S}\right) \rightarrow 0 .
$$

Proof. In the more general setting of a Galois extension of number fields $E / F$, with Galois group $G$, Kahn's Theorem 5.1 of [7] leads to an exact sequence induced by the transfer map and involving the number $r_{\infty}(E / F)$ of infinite primes of $F$ which ramify in $E$ (see [9, Prop. 1.6] or [12, Thm. 3.4]):

$$
0 \rightarrow K_{2}\left(\mathcal{O}_{E}^{S}\right)_{G} \rightarrow K_{2}\left(\mathcal{O}_{F}^{S}\right) \rightarrow\{ \pm 1\}^{r_{\infty}(E / F)} \rightarrow 0 .
$$

In our situation with $G=\langle\tau\rangle$ cyclic and $E$ totally real, $K_{2}\left(\mathcal{O}_{E}^{S}\right)_{G}=$ $K_{2}\left(\mathcal{O}_{E}^{S}\right) / K_{2}\left(\mathcal{O}_{E}^{S}\right)^{1-\tau}$ and $r_{\infty}(E / F)=0$. The result follows.

Proposition 2.2. $\left|K_{2}\left(\mathcal{O}_{E}^{S}\right)^{G}\right|=\left|K_{2}\left(\mathcal{O}_{F}^{S}\right)\right|$.

Proof. Theorem 2.1 gives $\left|K_{2}\left(\mathcal{O}_{F}^{S}\right)\right|=\left|K_{2}\left(\mathcal{O}_{E}^{S}\right)\right| /\left|K_{2}\left(\mathcal{O}_{E}^{S}\right)^{1-\tau}\right|$, while the exact sequence

$$
0 \rightarrow K_{2}\left(\mathcal{O}_{E}^{S}\right)^{G} \rightarrow K_{2}\left(\mathcal{O}_{E}^{S}\right) \stackrel{1-\tau}{\longrightarrow} K_{2}\left(\mathcal{O}_{E}^{S}\right)^{1-\tau} \rightarrow 0
$$

gives the same value for $\left|K_{2}\left(\mathcal{O}_{E}^{S}\right)^{G}\right|$. 
Let $\mathbb{Q}_{r}$ denote the $r$ th layer of the cyclotomic $\mathbb{Z}_{2}$-extension of $\mathbb{Q}$; it may be defined as $\mathbb{Q}_{r}=\mathbb{Q}\left(\mu_{2^{r+2}}\right)^{+}$, the maximal real subfield of the field of $2^{r+2}$ th roots of unity. Thus $\mathbb{Q}_{0}=\mathbb{Q}$ and $\mathbb{Q}_{1}=\mathbb{Q}(\sqrt{2})$. Also let $\mathbb{Q}_{\infty}$ denote the union of the $\mathbb{Q}_{r}$ for all natural numbers $r$. This is the cyclotomic $\mathbb{Z}_{2}$-extension of $\mathbb{Q}$. The cyclotomic $\mathbb{Z}_{2}$-extension of a field $L$ is $L_{\infty}=L \cdot \mathbb{Q}_{\infty}$, and the $n$th layer of this extension $L_{n}$ is the unique subfield of $L_{\infty}$ of degree $2^{n}$ over $L$.

Proposition 2.3. The cohomology groups

$$
H^{1}\left(G, K_{2}\left(\mathcal{O}_{E}^{S}\right)\right)=K_{2}\left(\mathcal{O}_{E}^{S}\right)^{-} / K_{2}\left(\mathcal{O}_{E}^{S}\right)^{1-\tau}
$$

and

$$
H^{2}\left(G, K_{2}\left(\mathcal{O}_{E}^{S}\right)\right)=K_{2}\left(\mathcal{O}_{E}^{S}\right)^{G} / K_{2}\left(\mathcal{O}_{E}^{S}\right)^{1+\tau}
$$

both have order 1 if $E=F_{1}$ is the first layer in the cyclotomic $\mathbb{Z}_{2}$-extension of $F$, and both have order 2 otherwise.

Proof. Since $K_{2}\left(\mathcal{O}_{E}^{S}\right)$ is finite and $G$ is cyclic, it follows that both cohomology groups have the same order (see [1, Prop. 11]). To compute the order of $H^{2}\left(G, K_{2}\left(\mathcal{O}_{E}^{S}\right)\right)=K_{2}\left(\mathcal{O}_{E}^{S}\right)^{G} / K_{2}\left(\mathcal{O}_{E}^{S}\right)^{1+\tau}$, view it as the cokernel of the map $K_{2}\left(\mathcal{O}_{E}^{S}\right) \stackrel{1+\tau}{\longrightarrow} K_{2}\left(\mathcal{O}_{E}^{S}\right)^{G}$. A standard functorial property states that this map factors as the transfer map $K_{2}\left(\mathcal{O}_{E}^{S}\right) \stackrel{\text { Trans }}{\longrightarrow} K_{2}\left(\mathcal{O}_{F}^{S}\right)$ followed by the map $K_{2}\left(\mathcal{O}_{F}^{S}\right) \stackrel{\iota *}{\rightarrow} K_{2}\left(\mathcal{O}_{E}^{S}\right)^{G}$ induced by the inclusion $\mathcal{O}_{F}^{S} \stackrel{\iota}{\rightarrow} \mathcal{O}_{E}^{S}$ of rings. Since the transfer is surjective, by Theorem 2.1, we are reduced to computing the order of the cokernel of $\iota_{*}$. By Proposition 2.2, the domain and codomain of this map have the same order, and thus the order of the cokernel equals the order of the kernel. The order of $\operatorname{ker}\left(\iota_{*}\right)$ is computed in [12, Lemma 7.3], yielding 1 if $E=F_{1}$ and 2 otherwise.

3. Cohomology of $W_{2}(E)$. We begin with two simple lemmas.

Lemma 3.1. If $L$ is a real field, and $L \cap \mathbb{Q}_{\infty}=\mathbb{Q}_{r}$, then $2^{r+3}$ exactly divides $w_{2}(L)$. In particular, $w_{2}(L)$ is always divisible by 8 .

Proof. This follows from the fact that $L\left(\mu_{2^{r+2+k}}\right)$ is the composite of the cyclic extensions $L\left(\mu_{4}\right)$ and $L \cdot \mathbb{Q}_{r+k}$. See [12, Lemma 7.2] for full details.

Lemma 3.2. Define the positive integers $r$ and $s$ by $F \cap \mathbb{Q}_{\infty}=\mathbb{Q}_{r}$, and $E \cap \mathbb{Q}_{\infty}=\mathbb{Q}_{s}$. Then $s>r$ if and only if $E=F_{1}$, the first layer of the cyclotomic $\mathbb{Z}_{2}$-extension of $F$. (In fact, $s \leq r+1$ always holds.)

Proof. We have $s>r$ if and only if $E \supset \mathbb{Q}_{r+1} \not \subset F$ if and only if $E \supset F \cdot \mathbb{Q}_{r+1}=F_{1} \supset F$. Since $[E: F]=2=\left[F_{1}: F\right]$, this last condition is equivalent to $E=F_{1}$.

For any prime number $p$, let $\mathbb{Z}_{(p)} \subset \mathbb{Q}$ denote the localization of $\mathbb{Z}$ at the prime ideal $(p)$. If $M$ is a $\mathbb{Z}$-module, then similarly let $M_{(p)} \cong M \otimes_{\mathbb{Z}} \mathbb{Z}_{(p)}$ be the localization of $M$ at $(p)$. Note that if $M$ is a finite abelian group, 
we may identify $M_{(p)}$ with the Sylow $p$-subgroup of $M$, which we call the p-part of $M$. If $M$ is a $\mathbb{Z}[G]$-module, then $M_{(p)}$ becomes a $\mathbb{Z}_{(p)}[G]$-module. If $I$ is an ideal of $\mathcal{R}=\mathbb{Z}[G]$, we may identify $I_{(p)}$ with the extended ideal $I \mathbb{Z}_{(p)}[G] \subset \mathbb{Z}_{(p)}[G]=\mathcal{R}_{(p)} \subset \mathbb{Q}[G]$.

Corollary 3.3. We have $W_{2}(E)_{(2)}=W_{2}(F)_{(2)}$ if and only if $E \neq F_{1}$.

Proof. Since $W_{2}(E)$ is cyclic and contains $W_{2}(F)$, this follows upon applying the preceding two lemmas.

Proposition 3.4. The cohomology groups

$$
H^{1}\left(G, W_{2}(E)\right)=W_{2}(E)^{-} / W_{2}(E)^{1-\tau}
$$

and

$$
H^{2}\left(G, W_{2}(E)\right)=W_{2}(E)^{G} / W_{2}(E)^{1+\tau}=W_{2}(F) / W_{2}(E)^{1+\tau}
$$

have order 1 if $E=F_{1}$ is the first layer in the cyclotomic $\mathbb{Z}_{2}$-extension of $F$, and order 2 otherwise.

Proof. Since $|G|=2$, and $W_{2}(E)$ is finite, the two cohomology groups have exponent 2 and equal orders. We will compute $H^{2}\left(G, W_{2}(E)\right)$; it suffices to consider the cohomology of $W_{2}(E)_{(2)}$.

According to Lemma 3.1, we may write $w_{2}(F)=8 \mathrm{~m}$. The action of $\tau$ on the cyclic group $W_{2}(E)$ fixes $W_{2}(F)$. Hence $\tau$ must act as $1+k w_{2}(F)$ for some integer $k$. Thus $W_{2}(E)_{(2)}^{1+\tau}=W_{2}(E)_{(2)}^{2+k w_{2}(F)}=\left(W_{2}(E)_{(2)}^{1+4 m}\right)^{2}=$ $W_{2}(E)_{(2)}^{2}$. Since $W_{2}(E)^{G}=W_{2}(F)$, we conclude that $H^{2}\left(G, W_{2}(E)_{(2)}\right)=$ $W_{2}(F)_{(2)} / W_{2}(E)_{(2)}^{2}$. Again using the cyclicity of $W_{2}(E)$, we see that this cohomology group is cyclic of exponent 2 and is non-trivial if and only if $W_{2}(E)_{(2)}=W_{2}(F)_{(2)}$. By the corollary, this is equivalent to $E \neq F_{1}$. (Here we also see that $s \leq r+1$.) Thus the cohomology groups are of order 2 when $E \neq F_{1}$, and are trivial otherwise.

4. The annihilator of $W_{2}(E)$. We have observed that $\tau$ acts on $W_{2}(E)$ as $N=1+k w_{2}(F)$ for some integer $k$. Thus $\tau-N$ and $\left|W_{2}(E)\right|$ lie in $\operatorname{Ann}_{\mathcal{R}}\left(W_{2}(E)\right)$. It is easy to see that these two elements generate $\operatorname{Ann}_{\mathcal{R}}\left(W_{2}(E)\right)$.

For future purposes, we would like to express $\operatorname{Ann}_{\mathcal{R}}\left(W_{2}(E)\right)$ in terms of $1+\tau$ and $1-\tau$. Let $e_{+}$and $e_{-}$denote the idempotents $(1+\tau) / 2$ and $(1-\tau) / 2$, respectively.

Proposition 4.1. $\operatorname{Ann}_{\mathcal{R}}\left(W_{2}(E)\right)$ is generated by $\left|W_{2}(E)^{1+\tau}\right|(1+\tau)$ and $\left|W_{2}(E)^{1-\tau}\right|(1-\tau)$ when $E \neq F_{1}$. It is generated by $\left|W_{2}(E)^{1+\tau}\right| e_{+}+$ $\left|W_{2}(E)^{1-\tau}\right| e_{-}$when $E=F_{1}$.

Proof. Note that $1-\tau$ induces an isomorphism from $W_{2}(E) / W_{2}(F)$ to $W_{2}(E)^{1-\tau}$. Thus from Corollary 3.3, we have $E=F_{1}$ if and only if 2 divides 
$\left|W_{2}(E) / W_{2}(F)\right|$, which holds if and only if 2 divides $\left|W_{2}(E)^{1-\tau}\right|$. Also note that the greatest common divisor of the orders of the subgroups $W_{2}(E)^{1+\tau}$ and $W_{2}(E)^{1-\tau}$ of the cyclic group $W_{2}(E)$ is the order of their intersection. This intersection clearly has exponent 2 and is cyclic. From Lemma 3.1 and Proposition 3.4, we know that the first group $W_{2}(E)^{1+\tau}$ has order divisible by 4 . We conclude that this greatest common divisor is 2 if $E=F_{1}$ and 1 otherwise.

The elements $\alpha^{+}=\left|W_{2}(E)^{1+\tau}\right|(1+\tau)$ and $\alpha^{-}=\left|W_{2}(E)^{1-\tau}\right|(1-\tau)$ clearly annihilate $W_{2}(E)$. When $E \neq F_{1}$, we show that these generate $\left|W_{2}(E)\right|$ and $\tau-N$ for some $N$, which in turn generate $\operatorname{Ann}_{\mathcal{R}}\left(W_{2}(E)\right)$. In this case, the integers $\left|W_{2}(E)^{1+\tau}\right|$ and $\left|W_{2}(E)^{1-\tau}\right|$ are relatively prime, so an integer linear combination of $\alpha^{+}$and $\alpha^{-}$yields the desired element of the form $\tau-N$. Also, $\left|W_{2}(E)\right| / 2$ is a common multiple of $\left|W_{2}(E)^{1+\tau}\right|$ and $\left|W_{2}(E)^{1-\tau}\right|$, as $\left|W_{2}(E)\right| /\left|W_{2}(E)^{1+\tau}\right|=\left|W_{2}(E)^{-}\right|$where $-1 \in W_{2}(E)^{-}$, while $\left|W_{2}(E)\right| /\left|W_{2}(E)^{1-\tau}\right|=\left|W_{2}(E)^{G}\right|=\left|W_{2}(F)\right|=8 m$ by Lemma 3.1 again. So $\alpha^{+}$and $\alpha^{-}$generate $\left(\left|W_{2}(E)\right| / 2\right)(1+\tau+1-\tau)=\left|W_{2}(E)\right|$.

Now suppose that $E=F_{1}$. We first show that $\gamma=\left|W_{2}(E)^{1+\tau}\right| e_{+}+$ $\left|W_{2}(E)^{1-\tau}\right| e_{-}=a e_{+}+b e_{-} \in \operatorname{Ann}_{\mathcal{R}}\left(W_{2}(E)\right)$. We have seen that the greatest common divisor of the two orders $a$ and $b$ here is 2 . If $\omega=\omega_{1}^{2} \in W_{2}(E)^{2}$, then $\omega^{\gamma}=\left(\omega_{1}^{1+\tau}\right)^{\left|W_{2}(E)^{1+\tau}\right|}\left(\omega_{1}^{1-\tau}\right)^{\left|W_{2}(E)^{1-\tau}\right|}=1$. On the other hand, if $\omega \notin$ $W_{2}(E)^{2}$, then its image generates $W_{2}(E) / W_{2}(E)^{2}$, and thus the image of $\omega^{1+\tau}$ generates $W_{2}(E)^{1+\tau} /\left(W_{2}(E)^{1+\tau}\right)^{2}$, and finally $\omega^{\left|W_{2}(E)^{1+\tau}\right| e_{+}}$generates $W_{2}(E)^{(1+\tau)\left|W_{2}(E)^{1+\tau}\right| / 2}$, which is cyclic of order 2 and therefore equals $\{-1\}$. Similarly, $\omega^{\left|W_{2}(E)^{1-\tau}\right| e_{-}}=-1$. Thus $\omega^{\gamma}=-1 \cdot-1=1$. Next we show that $\gamma$ generates both $\tau-N$ for some $N$, and $\left|W_{2}(E)\right|$. Since $\operatorname{gcd}(a / 2, b / 2)=1$ and $b / 2$ is odd while $a / 2$ is even, we have $\operatorname{gcd}(a, a / 2-b / 2)=1$. This shows that $\tau-N$ for some $N$ is an integer linear combination of $(1+\tau) \gamma=a \tau+a$ and $\gamma=(a / 2-b / 2) \tau+(a / 2+b / 2)$. Also, $\left|W_{2}(E)\right| / 2$ is a common multiple of $a$ and $b$, as seen above, and hence $\left|W_{2}(E)\right|=\left|W_{2}(E)\right|(1+\tau+1-\tau) / 2$ is generated by $\gamma(1+\tau)=a(1+\tau)$ and $\gamma(1-\tau)=b(1-\tau)$.

5. Properties of Fitting ideals. The following proposition lists the standard properties of Fitting ideals which we will need. Suppose that $A$ is a commutative Noetherian ring and $M$ is a finitely generated $A$-module. We denote the Fitting ideal of $M$ over $A$ by $\operatorname{Fit}_{A}(M)$. It is the ideal of $A$ generated by the determinants of all square matrices representing relations among a set of generators of $M$.

PROPOSITION 5.1.

(1) If $M$ is a cyclic A-module, then $\operatorname{Fit}_{A}(M)=\operatorname{Ann}_{A}(M)$.

(2) Given a morphism of commutative Noetherian rings $f: A \rightarrow B$, we have $\operatorname{Fit}_{B}\left(M \otimes_{A} B\right)=f\left(\operatorname{Fit}_{A}(M)\right) B$. In particular: 
(a) If $A \subset B$, then $\operatorname{Fit}_{B}\left(M \otimes_{A} B\right)=\operatorname{Fit}_{A}(M) B$.

(b) If $I$ is an ideal of $A$, then $\operatorname{Fit}_{A / I}(M / I M)=\left(\operatorname{Fit}_{A}(M)+I\right) / I$.

(c) If $T$ is a multiplicatively closed set in $A$ and $A_{T}=T^{-1} A$ (resp. $T^{-1} M=M_{T}$ ) is the corresponding ring (resp. module) of fractions, then $\operatorname{Fit}_{A_{T}}\left(M_{T}\right)=\left(\operatorname{Fit}_{A}(M)\right) A_{T}$.

(3) If $A=\mathbb{Z}$ and $M$ is finite, then $\operatorname{Fit}_{\mathbb{Z}}(M)=|M| \mathbb{Z}$.

(4) If $A=A_{1} \oplus A_{2}$ and correspondingly $M=M_{1} \oplus M_{2}$, then $\operatorname{Fit}_{A}(M)=$ $\operatorname{Fit}_{A_{1}}\left(M_{1}\right) \oplus \operatorname{Fit}_{A_{2}}\left(M_{2}\right)$.

Proof. Proofs of parts (1)-(3) may be found in [3]. The proof of part (4), and indeed every part, is a straightforward exercise.

We will also use a more specialized result on Fitting ideals, variations of which are found in [5] and [13].

Proposition 5.2. Suppose that $G$ is a finite abelian group and that $M$ is a finite $\mathbb{Z}[G]$-module. If $M$ is cohomologically trivial, then $\operatorname{Fit}_{\mathbb{Z}_{(p)}[G]}\left(M_{(p)}\right)$ is principal for any prime $p$.

Proof. See [2, Prop. 4]. The proof is based on the standard fact [1, Theorem 9$]$ that $M$ is cohomologically trivial if and only if its projective dimension as a $\mathbb{Z}[G]$-module is less than or equal to 1 .

6. Fitting ideals in the maximal order of $\mathbb{Q}[G]$. We will have occasion to extend ideals from $\mathcal{R}=\mathbb{Z}[G]=\mathbb{Z} \oplus \mathbb{Z} \tau$ to $\mathcal{S}=\mathbb{Z} e_{+} \oplus \mathbb{Z} e_{-}$, the maximal order in $\mathbb{Q}[G]$. Note that $\mathcal{S} \cong \mathcal{R} /(1-\tau) \oplus \mathcal{R} /(1+\tau)$ under the obvious homomorphism, the inverse homomorphism being $\left(\bar{r}_{1}, \bar{r}_{2}\right) \mapsto r_{1} e_{+}+r_{2} e_{-}$. Also, $\mathbb{Z} \cong \mathcal{R} /(1 \pm \tau)$ via the homomorphism sending each element of $\mathbb{Z}$ to its coset. We use these isomorphisms to make identifications in the next lemma.

Lemma 6.1. Suppose that $M$ is a finite $\mathcal{R}$-module. Then the $\mathcal{S}$-ideal $\operatorname{Fit}_{\mathcal{R}}(M) \mathcal{S}$ is generated by $\left|M^{G}\right| e_{+}+\left|M^{-}\right| e_{-}$.

Proof. Note that $\left|M^{G}\right|=\left|M / M^{1-\tau}\right|$ since $M / M^{G} \cong M^{1-\tau}$, and similarly $\left|M^{-}\right|=\left|M / M^{1+\tau}\right|$. Using part (2)(a) of Proposition 5.1, then part (4), and then part (3), we get

$$
\begin{aligned}
\operatorname{Fit}_{\mathcal{R}}(M) \mathcal{S} & =\operatorname{Fit}_{\mathcal{S}}\left(M \otimes_{\mathcal{R}} \mathcal{S}\right)=\operatorname{Fit}_{\mathcal{R} /(1-\tau) \oplus \mathcal{R} /(1+\tau)}\left(M / M^{1-\tau} \oplus M / M^{1+\tau}\right) \\
& =\operatorname{Fit}_{\mathcal{R} /(1-\tau)}\left(M / M^{1-\tau}\right) \oplus \operatorname{Fit}_{\mathcal{R} /(1+\tau)}\left(M / M^{1+\tau}\right) \\
& =\left(\left|M / M^{1-\tau}\right| e_{+}+\left|M / M^{1+\tau}\right| e_{-}\right) \mathcal{S} \\
& =\left(\left|M^{G}\right| e_{+}+\left|M^{-}\right| e_{-}\right) \mathcal{S} .
\end{aligned}
$$

Let $k^{+}=\left|K_{2}\left(\mathcal{O}_{E}^{S}\right)^{G}\right|$ (which equals $\left|K_{2}\left(\mathcal{O}_{F}^{S}\right)\right|$ by Proposition 2.2), and $k^{-}=\left|K_{2}\left(\mathcal{O}_{E}^{S}\right)^{-}\right|$. Similarly let $w^{+}=\left|W_{2}(E)^{G}\right|=\left|W_{2}(F)\right|$ and $w^{-}=$ $\left|W_{2}(E)^{-}\right|$. 
Proposition 6.2. We have

$$
\begin{aligned}
\mathcal{I}^{\mathrm{Fit}} \mathcal{S} & =\left(k^{+} e_{+}+k^{-} e_{-}\right) \mathcal{S}, \\
\operatorname{Ann}_{\mathcal{R}}\left(W_{2}(E)\right) \mathcal{S} & =\operatorname{Fit}_{\mathcal{R}}\left(W_{2}(E)\right) \mathcal{S}=\left(w^{+} e_{+}+w^{-} e_{-}\right) \mathcal{S} .
\end{aligned}
$$

Proof. Apply the lemma to $K_{2}\left(\mathcal{O}_{E}^{S}\right)$ and $W_{2}(E)$, and use part (1) of Proposition 5.1.

Our goal of course is to compute $\mathcal{I}^{\text {Fit }}=\operatorname{Fit}_{\mathcal{R}}\left(K_{2}\left(\mathcal{O}_{E}^{S}\right)\right)$; we will also need $\operatorname{Ann}_{\mathcal{R}}\left(W_{2}(E)\right)=\operatorname{Fit}_{\mathcal{R}}\left(W_{2}(E)\right)$. Proposition 6.2 will help us identify the former. The latter is known and easily done directly. We will derive it in a form most useful to us in the next section. That, combined with Proposition 6.2 and the evaluation of $\theta_{E / F}^{S}(-1)$, will be enough to provide the odd part of our main result (Theorem 1.3). To obtain the 2-part of Theorem 1.3, we will explicitly compute a key element of $\mathcal{I}^{\text {Fit }}$ in Section 9.

7. The Stickelberger ideal $\mathcal{I}^{\mathrm{Sti}}=\operatorname{Ann}_{\mathcal{R}}\left(W_{2}(E)\right) \theta_{E / F}^{S}(-1)$. Associated with each character $\chi$ of $G$, we have the Artin $L$-function with Euler factors for primes in $S$ removed:

$$
L_{E / F}^{S}(s, \chi)=\sum_{\substack{\mathfrak{a} \text { integral } \\(\mathfrak{a}, S)=1}} \frac{\chi\left(\sigma_{\mathfrak{a}}\right)}{\mathrm{Na}^{s}}=\prod_{\text {prime } \mathfrak{p} \notin S}\left(1-\frac{\chi\left(\sigma_{\mathfrak{p}}\right)}{\mathrm{Np}^{s}}\right)^{-1} .
$$

These are related to the equivariant $L$-function by

$$
\theta_{E / F}^{S}(s)=\sum_{\chi} L_{E / F}^{S}(s, \bar{\chi}) e_{\chi}
$$

where $\bar{\chi}$ is the complex conjugate of $\chi$, as the two sides of this equation agree after multiplication by each idempotent $e_{\chi}=|G|^{-1} \sum_{\sigma \in G} \chi(\sigma) \sigma^{-1}$. In our case we just have the trivial character $\chi_{0}$ and the non-trivial character $\chi_{1}$, giving

$$
\theta_{E / F}^{S}(s)=L_{E / F}^{S}\left(s, \chi_{0}\right) e_{+}+L_{E / F}^{S}\left(s, \chi_{1}\right) e_{-}=\zeta_{F}^{S}(s) e_{+}+\frac{\zeta_{E}^{S}(s)}{\zeta_{F}^{S}(s)} e_{-},
$$

by standard properties of Artin $L$-functions. Thus

$$
\theta_{E / F}^{S}(-1)=\zeta_{F}^{S}(-1) e_{+}+\frac{\zeta_{E}^{S}(-1)}{\zeta_{F}^{S}(-1)} e_{-} .
$$

Referring now to Conjecture 1.1 and Theorem 1.2, we clearly obtain the following.

Proposition 7.1. Assuming the Birch-Tate conjecture for $E$ and for $F$, we have

$$
(-1)^{|S|} \theta_{E / F}^{S}(-1)=\frac{\left|K_{2}\left(\mathcal{O}_{F}^{S}\right)\right|}{w_{2}(F)} e_{+}+(-1)^{\left|S_{E}\right|} \frac{w_{2}(F)}{w_{2}(E)} \frac{\left|K_{2}\left(\mathcal{O}_{E}^{S}\right)\right|}{\left|K_{2}\left(\mathcal{O}_{F}^{S}\right)\right|} e_{-} .
$$

Equality of the odd parts holds unconditionally. 
Lemma 7.2. Assuming the Birch-Tate conjecture for $E$ and $F$, we have $w^{+} e_{+} \theta_{E / F}^{S}(-1)= \pm k^{+} e_{+}$and $w^{-} e_{-} \theta_{E / F}^{S}(-1)= \pm k^{-} e_{-}$. Equality of odd parts holds unconditionally.

Proof. Using Proposition 7.1, we see that

$$
\pm w^{+} e_{+} \theta_{E / F}^{S}(-1)=\left(w^{+} e_{+}\right)\left(\frac{k^{+}}{w^{+}} e_{+} \pm \frac{w^{+}}{w_{2}(E)} \frac{\left|K_{2}\left(\mathcal{O}_{E}^{S}\right)\right|}{k^{+}} e_{-}\right)=k^{+} e_{+} .
$$

Similarly,

$$
\begin{aligned}
\pm w^{-} e_{-} \theta_{E / F}^{S}(-1) & =\left(w^{-} e_{-}\right)\left(\frac{k^{+}}{w^{+}} e_{+} \pm \frac{w^{+}}{w_{2}(E)} \frac{\left|K_{2}\left(\mathcal{O}_{E}^{S}\right)\right|}{k^{+}} e_{-}\right) \\
& = \pm \frac{w^{+} w^{-}}{w_{2}(E)} \frac{\left|K_{2}\left(\mathcal{O}_{E}^{S}\right)\right|}{k^{+}} e_{-} .
\end{aligned}
$$

Here $w_{2}(E) / w^{-}=\left|W_{2}(E)^{1+\tau}\right|$, so

$$
\frac{w^{+} w^{-}}{w_{2}(E)}=\left|W_{2}(E)^{G}\right| /\left|W_{2}(E)^{1+\tau}\right|=\left|K_{2}\left(\mathcal{O}_{E}^{S}\right)^{G}\right| /\left|K_{2}\left(\mathcal{O}_{E}^{S}\right)^{1+\tau}\right|,
$$

by Propositions 3.4 and 2.3. By Proposition 2.2, we also have $\left|K_{2}\left(\mathcal{O}_{E}^{S}\right)^{G}\right|=$ $\left|K_{2}\left(\mathcal{O}_{F}^{S}\right)\right|=k^{+}$. Making these substitutions above leads to the equality

$$
\pm\left(w^{-} e_{-}\right) \theta_{E / F}^{S}(-1)=\left(\left|K_{2}\left(\mathcal{O}_{E}^{S}\right)\right| /\left|K_{2}\left(\mathcal{O}_{E}^{S}\right)^{1+\tau}\right|\right) e_{-}=k^{-} e_{-},
$$

as desired. This proof works for the odd parts without assuming the BirchTate conjecture.

Proposition 7.3. Assuming the Birch-Tate conjecture for $E$ and $F$, we have $\mathcal{I}^{\text {Sti }} \mathcal{S}=\mathcal{I}^{\text {Fit }} \mathcal{S}$. The equality $\mathcal{I}^{\text {Sti }} \mathcal{S}[1 / 2]=\mathcal{I}^{\text {Fit }} \mathcal{S}[1 / 2]$ holds unconditionally.

Proof. From Proposition 6.2 we see that $\mathcal{I}^{\text {Fit }} \mathcal{S}$ is generated by $k^{+} e_{+}+$ $k^{-} e_{-}$as an ideal in $\mathcal{S}$. Similarly, $\mathcal{I}^{\mathrm{Sti}} \mathcal{S}=\operatorname{Ann}_{\mathcal{R}}\left(W_{2}(E)\right) \theta_{E / F}^{S}(-1) \mathcal{S}$ is generated by $\left(w^{+} e_{+}+w^{-} e_{-}\right) \theta_{E / F}^{S}(-1)$. Thus it suffices to show that $k^{+} e_{+}+k^{-} e_{-}$ and $\left(w^{+} e_{+}+w^{-} e_{-}\right) \theta_{E / F}^{S}(-1)$ are associates in $\mathcal{S}$. This is easily accomplished upon multiplying by one of the units \pm 1 or $\pm \tau$, and using Lemma 7.2. If we first extend ideals to $\mathcal{S}[1 / 2]$, the same proof works unconditionally.

Corollary 7.4. $\mathcal{I}^{\text {Fit }} \mathcal{R}[1 / 2]=\mathcal{I}^{\text {Sti }} \mathcal{R}[1 / 2]$. Consequently, $\mathcal{I}_{(p)}^{\text {Fit }}=\mathcal{I}_{(p)}^{\text {Sti }}$ for all odd primes $p$.

Proof. Noting that $\mathcal{R}[1 / 2]=\mathcal{S}[1 / 2]$, we see that the first equality is just a restatement of the unconditional part of Proposition 7.3. The second equality follows upon extending ideals from $\mathcal{R}[1 / 2]=\mathbb{Z}[1 / 2][G]$ to $\mathcal{R}_{(p)}=\mathbb{Z}_{(p)}[G]$. 
Corollary 7.4 establishes the unconditional part of Theorem 1.3. Our goal now is to show that $\mathcal{I}_{(2)}^{\mathrm{Fit}}=\mathcal{I}_{(2)}^{\mathrm{Sti}}$, assuming the 2-part of the Birch-Tate conjecture.

8. Ideals in $\mathcal{R}_{(2)}=\mathbb{Z}_{(2)}[G]$. Since $G$ is a 2-group, $\mathcal{R}_{(2)}=\mathbb{Z}_{(2)}[G]$ is a local ring with maximal ideal $\mathfrak{m}=(2,1-\tau)=(1+\tau, 1-\tau)$.

We will need to consider the relationship between $\mathcal{R}_{(2)}$ and the overring $\mathcal{S}_{(2)}=\mathbb{Z}_{(2)} e_{+} \oplus \mathbb{Z}_{(2)} e_{-} \cong \mathbb{Z}_{(2)} \oplus \mathbb{Z}_{(2)}$, which is clearly a principal ideal ring.

LEMMA 8.1. The group of units $\mathcal{S}_{(2)}^{\times}$of $\mathcal{S}_{(2)}$ equals the group of units of $\mathcal{R}_{(2)}$.

Proof.

$$
\begin{aligned}
\mathcal{S}_{(2)}^{\times} & =\mathbb{Z}_{(2)}^{\times} e_{+} \oplus \mathbb{Z}_{(2)}^{\times} e_{-}=\left(1+2 \mathbb{Z}_{(2)}\right) e_{+} \oplus\left(1+2 \mathbb{Z}_{(2)}\right) e_{-} \\
& =\left(e_{+}+e_{-}\right)+\mathbb{Z}_{(2)}(1+\tau)+\mathbb{Z}_{(2)}(1-\tau)=1+\mathfrak{m}=\mathcal{R}_{(2)}^{\times} .
\end{aligned}
$$

Lemma 8.2. Suppose that $I$ is an ideal of finite index in $\mathcal{R}_{(2)}$. Let $\alpha$ be a generator in the principal ideal ring $\mathcal{S}_{(2)}$ for the extended ideal IS $\mathcal{S}_{(2)}$. Then $\alpha \in I$.

Proof. Modifying by units, we may assume that $\alpha=2^{i} e_{+}+2^{j} e_{-}$. Since $\mathcal{S}_{(2)}=\mathcal{R}_{(2)} e_{+} \oplus \mathcal{R}_{(2)} e_{-}$, we see that $2^{i} e_{+}+2^{j} e_{-}$generates $I \mathcal{S}_{(2)}=I e_{+} \oplus I e_{-}$. Thus $2^{i} e_{+}$generates $I e_{+}$over $\mathbb{Z}_{(2)}$ and $2^{j} e_{-}$generates $I e_{-}$. Consequently, $2^{i} e_{+}+2^{j^{\prime}} e_{-} \in I$ for some $j^{\prime} \geq j$ and $2^{i^{\prime}} e_{+}+2^{j} e_{-} \in I$ for some $i^{\prime} \geq i$. Multiplying the second element by $1-\tau \in \mathcal{R}_{(2)}$, we also find that $2^{j+1} e_{-} \in I$. If $j^{\prime}=j$, then we have $2^{i} e_{+}+2^{j} e_{-} \in I$ as desired. If not, then $2^{i} e_{+}+2^{j^{\prime}} e_{-} \in I$ and $2^{j+1} e_{-} \in I$ generate $2^{i} e_{+} \in I$. Combined with $2^{i^{\prime}} e_{+}+2^{j} e_{-} \in I$, this generates $2^{j} e_{-} \in I$, and finally $2^{i} e_{+}+2^{j} e_{-} \in I$.

REMARK 8.3. In the setting of Lemma 8.2, one can in fact show that $I$ is either the principal ideal generated by $\alpha$ or the non-principal ideal generated by $\alpha e_{+}$and $\alpha e_{-}$.

9. Computation of $\mathcal{I}_{(2)}^{\mathrm{Fit}}$. Assuming the 2-part of the Birch-Tate conjecture for $E$ and $F$, we now perform the computation of $\mathcal{I}_{(2)}^{\text {Fit }}$, separating it into two different cases.

Proposition 9.1. Suppose that $E=F_{1}$, and assume that the 2-part of the Birch-Tate conjecture holds for $F$ and for $E$. Then $\mathcal{I}_{(2)}^{\mathrm{Fit}}=\mathcal{I}_{(2)}^{\mathrm{Sti}}$.

Proof. Since $E=F_{1}$, Propositions 2.3 and 5.2 show that $\mathcal{I}_{(2)}^{\text {Fit }}$ is a principal ideal in $\mathcal{R}_{(2)}$. Let $\alpha \in \mathcal{R}_{(2)}$ be a generator for $\mathcal{I}_{(2)}^{\text {Fit }}$. Similarly, Propositions 3.4 and 5.2 show that $\operatorname{Ann}_{R}\left(W_{2}(E)\right) \mathcal{R}_{(2)}$ and hence $\mathcal{I}_{(2)}^{\text {Sti }}=$ 
$\theta_{E / F}^{S}(-1) \operatorname{Ann}_{R}\left(W_{2}(E)\right) \mathcal{R}_{(2)}$ is principal. We can also see this more explicitly from Proposition 4.1. Let $\beta \in \mathcal{R}_{(2)}$ be a generator for $\mathcal{I}_{(2)}^{\text {Sti }}$. From Proposition 7.3 , we deduce that $\mathcal{I}_{(2)}^{\text {Fit }}$ and $\mathcal{I}_{(2)}^{\text {Sti }}$ extend to the same ideal in $\mathcal{S}_{(2)}$. Thus $\alpha$ and $\beta$ are associates in $\mathcal{S}_{(2)}$. By Lemma 8.1, they are associates in $\mathcal{R}_{(2)}$, and thus $\mathcal{I}_{(2)}^{\mathrm{Fit}}=\mathcal{I}_{(2)}^{\mathrm{Sti}}$.

We now turn to the case of $E \neq F_{1}$, in which $\left|K_{2}\left(\mathcal{O}_{E}^{S}\right)^{-} / K_{2}\left(\mathcal{O}_{E}^{S}\right)^{1-\tau}\right|=2$ by Proposition 2.3. We will use a lemma to simplify the computation.

Lemma 9.2. Suppose that $M$ is a finite abelian 2-group, and $m \in M$ is an element of order 2 . Then $M$ is isomorphic to a direct product of nontrivial cyclic groups, one of which contains $m$.

Proof. This is a straightforward exercise.

Proposition 9.3. If $E \neq F_{1}$, then $k^{+} e_{+} \in \mathcal{I}_{(2)}^{\text {Fit }}$.

Proof. First, $\mathcal{I}_{(2)}^{\mathrm{Fit}}=\operatorname{Fit}_{\mathcal{R}_{(2)}}\left(K_{2}\left(\mathcal{O}_{E}^{S}\right)_{(2)}\right)$ by part (2)(c) of Proposition 5.1. We know by Proposition 2.3 that $K_{2}\left(\mathcal{O}_{E}^{S}\right)^{-} / K_{2}\left(\mathcal{O}_{E}^{S}\right)^{1-\tau}$ has order 2 , and it follows by decomposing $K_{2}\left(\mathcal{O}_{E}^{S}\right)$ into a 2-part and an odd part that $K_{2}\left(\mathcal{O}_{E}^{S}\right)_{(2)}^{-} / K_{2}\left(\mathcal{O}_{E}^{S}\right)_{(2)}^{1-\tau}$ has order 2 .

Now apply Lemma 9.2 with $M=K_{2}\left(\mathcal{O}_{E}^{S}\right)_{(2)} / K_{2}\left(\mathcal{O}_{E}^{S}\right)_{(2)}^{1-\tau}$ and $m$ as a generator of the subgroup $K_{2}\left(\mathcal{O}_{E}^{S}\right)_{(2)}^{-} / K_{2}\left(\mathcal{O}_{E}^{S}\right)_{(2)}^{1-\tau}$ of order 2 . Let $\bar{\gamma}_{i}, 1 \leq i \leq r$, be the generators of $K_{2}\left(\mathcal{O}_{E}^{S}\right)_{(2)} / K_{2}\left(\mathcal{O}_{E}^{S}\right)_{(2)}^{1-\tau}$ obtained from the lemma, with each $\gamma_{i} \in K_{2}\left(\mathcal{O}_{E}^{S}\right)_{(2)}$. Then $\bar{\gamma}_{i}$ has order $d_{i}=2^{c_{i}}$, a positive power of 2 for each $i$, and $\prod_{i} d_{i}=\left|K_{2}\left(\mathcal{O}_{E}^{S}\right)_{(2)} / K_{2}\left(\mathcal{O}_{E}^{S}\right)_{(2)}^{1-\tau}\right|$. Denote this order by $k_{(2)}^{+}$. Again the decomposition of $K_{2}\left(\mathcal{O}_{E}^{S}\right)$ into an odd part and a 2-part shows that $k_{(2)}^{+}$ is the 2-part of $\left|K_{2}\left(\mathcal{O}_{E}^{S}\right) / K_{2}\left(\mathcal{O}_{E}^{S}\right)^{1-\tau}\right|$, which equals $\left|K_{2}\left(\mathcal{O}_{F}^{S}\right)\right|=\left|K_{2}\left(\mathcal{O}_{E}^{S}\right)^{G}\right|$ $=k^{+}$by Theorem 2.1 and Proposition 2.2. Thus $k^{+}$and $k_{(2)}^{+}$are associates in $\mathbb{Z}_{(2)} \subset \mathcal{R}_{(2)}$ and it suffices to show that $k_{(2)}^{+} e_{+} \in \operatorname{Fit}_{\mathcal{R}_{(2)}}\left(K_{2}\left(\mathcal{O}_{E}^{S}\right)_{(2)}\right)$.

The elements $\bar{\gamma}_{i}$ for $i=1, \ldots, r$ generate $K_{2}\left(\mathcal{O}_{E}^{S}\right)_{(2)} / K_{2}\left(\mathcal{O}_{E}^{S}\right)_{(2)}^{1-\tau}$ as a group, hence as an $\mathcal{R}_{(2)}$-module. But $1-\tau \in \mathfrak{m}$, the maximal ideal of the local ring $\mathcal{R}_{(2)}$, and hence by Nakayama's lemma, the elements $\gamma_{i}$ for $i=1, \ldots, r$ generate $K_{2}\left(\mathcal{O}_{E}^{S}\right)_{(2)}$ as an $\mathcal{R}_{(2)}$-module. Finding $r$ relations among these $r$ generators will provide an element of the Fitting ideal in question by taking the determinant of the corresponding $r$-by- $r$ relations matrix.

The fact that the elements $\gamma_{i}$ for $i=1, \ldots, r$ generate $K_{2}\left(\mathcal{O}_{E}^{S}\right)_{(2)}$ over $\mathcal{R}_{(2)}$ implies that the elements $\gamma_{i}^{1-\tau}$ for $i=1, \ldots, r$ generate $K_{2}\left(\mathcal{O}_{E}^{S}\right)_{(2)}^{1-\tau}$. For each $i, \bar{\gamma}_{i}$ has order $d_{i}$, and this means that $\gamma_{i}^{d_{i}} \in K_{2}\left(\mathcal{O}_{E}^{S}\right)_{(2)}^{1-\tau}$. Multiplying by $\gamma_{i}^{(\tau-1) d_{i} / 2} \in K_{2}\left(\mathcal{O}_{E}^{S}\right)_{(2)}^{1-\tau}$ gives us $\gamma_{i}^{(1+\tau) d_{i} / 2} \in K_{2}\left(\mathcal{O}_{E}^{S}\right)_{(2)}^{1-\tau}$ for each $i$. As 
the elements $\gamma_{i}^{1-\tau}$ for $i=1, \ldots, r$ generate this last $\mathcal{R}_{(2)}$-module, we have, for each $i$,

$$
\gamma_{i}^{(1+\tau) d_{i} / 2}=\prod_{j=1}^{r}\left(\gamma_{j}^{1-\tau}\right)^{b_{i j}}
$$

for some elements $b_{i j} \in \mathcal{R}_{(2)}$. Let $D$ be the $r$-by- $r$ diagonal matrix of the $d_{i}$, and $B$ be the matrix of the $b_{i j}$. Then the last equation shows that $e_{+} D-$ $(1-\tau) B$ is a relations matrix for the generators $\gamma_{i}$ of $K_{2}\left(\mathcal{O}_{E}^{S}\right)_{(2)}$. Hence

$$
\delta=\operatorname{det}\left(e_{+} D-(1-\tau) B\right) \in \operatorname{Fit}_{\mathcal{R}_{(2)}}\left(K_{2}\left(\mathcal{O}_{E}^{S}\right)_{(2)}\right)=\mathcal{I}_{(2)}^{\mathrm{Fit}} .
$$

Modulo $1-\tau$, we have $\delta \equiv \operatorname{det}\left(e_{+} D\right)=\left(e_{+}\right)^{r} \operatorname{det}(D)=e_{+} \prod_{i} d_{i}=$ $e_{+} k_{(2)}^{+}$. Before considering $\delta$ modulo $1+\tau$, recall that the application of Lemma 9.2 also shows that $\bar{\gamma}_{t}^{d_{t} / 2}$ generates $K_{2}\left(\mathcal{O}_{E}^{S}\right)_{(2)}^{-} / K_{2}\left(\mathcal{O}_{E}^{S}\right)_{(2)}^{1-\tau}$ for some $t$. It follows that $\gamma_{t}^{d_{t} / 2} \in K_{2}\left(\mathcal{O}_{E}^{S}\right)_{(2)}^{-}$, and consequently $\gamma_{t}^{\left(d_{t} / 2\right)(1+\tau)}$ is trivial. Hence we may choose $b_{t j}=0$ for all $j$, making $\operatorname{det}(B)=0$. Now we see that $\delta \equiv \operatorname{det}(-2 B)=0$ modulo $1+\tau$. Thus $\delta-k_{(2)}^{+} e_{+} \in$ $(1+\tau) \cap(1-\tau)=(0)$ in $\mathcal{R}_{(2)}$. We conclude that $k_{(2)}^{+} e_{+}=\delta \in \mathcal{I}_{(2)}^{\text {Fit }}$, and we know that this implies the desired result.

Proposition 9.4. Suppose that $E \neq F_{1}$, and assume that the BirchTate conjecture holds for $F$ and $E$. Then $\mathcal{I}_{(2)}^{\text {Fit }}=\mathcal{I}_{(2)}^{\text {Sti }}$.

Proof. By Proposition 6.2, $k^{+} e_{+}+k^{-} e_{-}$generates $\mathcal{I}^{\text {Fit } \mathcal{S}}$ as an ideal of $\mathcal{S}$, and hence this same element generates the extended ideal $\mathcal{I}^{\text {Fit }} \mathcal{S}_{(2)}$ as an ideal of $\mathcal{S}_{(2)}$. Lemma 8.2 then implies that $k^{+} e_{+}+k^{-} e_{-} \in \mathcal{I}_{(2)}^{\text {Fit }}$. On the other hand, Proposition 9.3 gives $k^{+} e_{+} \in \mathcal{I}_{(2)}^{\text {Fit }}$. Subtracting, we see that both $k^{+} e_{+}$and $k^{-} e_{-}$lie in $\mathcal{I}_{(2)}^{\text {Fit }}$. Thus

$$
\begin{gathered}
\mathcal{I}_{(2)}^{\mathrm{Fit}} \supset k^{+} e_{+} \mathcal{R}_{(2)}+k^{-} e_{-} \mathcal{R}_{(2)}=\left(k^{+} e_{+}+k^{-} e_{-}\right)\left(\mathcal{R}_{(2)} e_{+}+\mathcal{R}_{(2)} e_{-}\right) \\
=\left(k^{+} e_{+}+k^{-} e_{-}\right) \mathcal{S}_{(2)}=\mathcal{I}_{(2)}^{\mathrm{Fit}} \mathcal{S}_{(2)} \supset \mathcal{I}_{(2)}^{\mathrm{Fit}},
\end{gathered}
$$

by Proposition 6.2 again. We conclude that $\mathcal{I}_{(2)}^{\text {Fit }}=k^{+} e_{+} \mathcal{R}_{(2)}+k^{-} e_{-} \mathcal{R}_{(2)}$.

Turning to $\mathcal{I}_{(2)}^{\mathrm{Sti}}$, we deduce from Proposition 4.1 that $\operatorname{Ann}_{\mathcal{R}}\left(W_{2}(E)\right)$ is generated by $\left|W_{2}(E)^{1+\tau}\right|(1+\tau)$ and $\left|W_{2}(E)^{1-\tau}\right|(1-\tau)$. By Proposition 2.3, these generators may also be written as $w^{+} e_{+}$and $w^{-} e_{-}$. Hence $\mathcal{I}^{\text {Sti }}=$ $\theta_{E / F}^{S}(-1) \operatorname{Ann}_{\mathcal{R}}\left(W_{2}(E)\right)$ is generated by $w^{+} e_{+} \theta_{E / F}^{S}(-1)$ and $w^{-} e_{-} \theta_{E / F}^{S}(-1)$. Using Lemma 7.2, we see that these generators equal $\pm k^{+} e_{+}$and $\pm k^{-} e_{-}$. Consequently, $\mathcal{I}^{\mathrm{Sti}}$ is generated by $k^{+} e_{+}$and $k^{-} e_{-}$, so

$$
\mathcal{I}_{(2)}^{\mathrm{Sti}}=k^{+} e_{+} \mathcal{R}_{(2)}+k^{-} e_{-} \mathcal{R}_{(2)}=\mathcal{I}_{(2)}^{\mathrm{Fit}} \text {. }
$$


Now we can complete the proof of Theorem 1.3. From Corollary 7.4 we get the unconditional statement in Theorem 1.3, and also

$$
\mathcal{I}_{(p)}^{\mathrm{Fit}}=\mathcal{I}_{(p)}^{\mathrm{Sti}} \subset \mathbb{Z}_{(p)}[G] \subset \mathbb{Q}[G]
$$

for each odd prime $p$. Under the Birch-Tate conjecture for $E$ and $F$, Propositions 9.1 and 9.4 show that $\mathcal{I}_{(2)}^{\text {Fit }}=\mathcal{I}_{(2)}^{\text {Sti }}$. Note that for any ideal $I$ of $\mathcal{R}$, the intersection over all rational primes $\bigcap_{p} I_{(p)}$ equals $I$, because for each $\alpha \in \bigcap_{p} I_{(p)}$, the $\mathbb{Z}$-ideal $\{a \in \mathbb{Z}: a \alpha \in I\}$ is contained in no prime ideal of $\mathbb{Z}$, hence equals $\mathbb{Z}$. Thus

$$
\mathcal{I}^{\mathrm{Fit}}=\bigcap_{p} \mathcal{I}_{(p)}^{\mathrm{Fit}}=\bigcap_{p} \mathcal{I}_{(p)}^{\mathrm{Sti}}=\mathcal{I}^{\mathrm{Sti}}
$$

Acknowledgements. We thank Cristian Popescu, David Solomon and Jason Price for helpful input on the subject matter and exposition of this paper. We also thank the Department of Mathematics at the University of California, San Diego for its hospitality during a 2006-2007 sabbatical year.

\section{References}

[1] M. F. Atiyah and C. T. C. Wall, Cohomology of groups, in: Algebraic Number Theory, J. W. S. Cassels and A. Fröhlich (eds.), Academic Press, 1967, 94-115.

[2] P. Cornacchia and C. Greither, Fitting ideals of class groups of real fields with prime power conductor, J. Number Theory 73 (1998), 459-471.

[3] D. Eisenbud, Commutative Algebra with a View Towards Algebraic Geometry, Grad. Texts in Math. 150, Springer, 1995.

[4] H. Garland, A finiteness theorem for $K_{2}$ of a number field, Ann. of Math. 94 (1971), $534-548$.

[5] C. Greither, Some cases of Brumer's conjecture for abelian CM extensions of totally real fields, Math. Z. 233 (2000), 515-534.

[6] -, Determining Fitting ideals of minus class groups via the equivariant Tamagawa number conjecture, Compos. Math. 143 (2007), 1399-1426.

[7] B. Kahn, Descente galoissienne et $K_{2}$ des corps de nombres, K-Theory 7 (1993), $55-100$.

[8] M. Kolster, Cohomological version of the Lichtenbaum conjecture at the prime 2, appendix to: J. Rognes and C. Weibel, Two-primary algebraic K-theory of rings of integers in number fields, J. Amer. Math. Soc. 13 (2000), 1-54.

[9] M. Kolster and A. Movahhedi, Galois co-descent for étale wild kernels and capitulation, Ann. Inst. Fourier (Grenoble) 50 (2000), 35-65.

[10] B. Mazur and A. Wiles, Class fields of abelian extensions of $\mathbb{Q}$, Invent. Math. 76 (1984), 179-330.

[11] D. Quillen, Finite generation of the groups $K_{i}$ of rings of algebraic integers, in: Algebraic K-theory I, H. Bass (ed.), Lecture Notes in Math. 341, Springer, 1973, 179-198.

[12] J. W. Sands and L. D. Simons, Values at $s=-1$ of L-functions for multi-quadratic extensions of number fields and annihilation of the tame kernel, J. London Math. Soc. 76 (2007), 545-555. 
[13] R. Schoof, Minus class groups of the fields of the lth roots of unity, Math. Comp. 67 (1998), 1225-1245.

[14] J. T. Tate, Symbols in Arithmetic, in: Actes, Congrès Intern. Math. (Nice, 1970), tome 1, Gauthier-Villars, 1971, 201-211.

[15] A. Wiles, The Iwasawa conjecture for totally real fields, Ann. of Math. 131 (1990), $493-540$.

Department of Mathematics and Statistics

University of Vermont

Burlington, VT 05401, U.S.A.

E-mail: Jonathan.Sands@uvm.edu

Received on 12.9.2007

and in revised form on 20.11.2007 